\title{
Breast Cancer Screening Rates Among Women Aged 20 Years and Above - China, 2015
}

\author{
Mei Zhang, ${ }^{1,2}$; Yijing Zhong, ${ }^{2,28}$; Heling Bao ${ }^{1,3}$; Zhenping Zhao'; Zhengjing Huangi'; Xiao Zhang'; \\ Chun Li'; Maigeng Zhou ${ }^{1, \alpha}$; Limin Wang'; Jing Wu'; Xiaoying Zheng ${ }^{2, * ;}$; Linhong Wang ${ }^{1, * \#}$
}

\section{Summary \\ What is already known about this topic?}

Breast cancer is the most common cancer in women in China and around the world. By 2019, 121 countries have instituted a national screening program as a secondary prevention measure for breast cancer.

What is added by this report?

Breast cancer screening rates in China were $18.9 \%$ in women aged 20 years and above, and $25.7 \%$ in women aged 35-64 years in 2015. The screening rate for women aged 20 years and above was significantly higher in urban areas than in rural areas $(24.6 \%$ vs. $15.0 \%)$, and in the eastern region than in the central and western regions $(24.0 \%$ vs. $15.1 \%$ and $15.3 \%)$.

What are the implications for public health practice?

Continued efforts should be made to strengthen national and local policy initiatives and financial support for population-based, organized screening programs for breast cancer. Health education and accessibility of screening services to women across the country should be strengthened, especially for women aged 50 years and above.

Breast cancer is the most common cancer in women in China and around the world. Age-standardized incidence and mortality by Chinese standard population were $31.54 / 100,000$ population and 6.67/100,000, respectively, in China in 2015 (1). The breast cancer screening rate in China reached $21.7 \%$ in women aged 18 years and above in 2010 (2) and $22.5 \%$ in women aged 35-64 years in 2013 (3). To understand the latest screening coverage in China, this study estimated screening rates in 2015 based on nationally and provincially-representative survey data, and Rao Scott chi-squared tests and logistic regression models were conducted to analyze key demographic and geographic factors. This study showed improving but still low levels of breast cancer screening coverage of women in China. Continued efforts should be made to strengthen national and local policy initiatives and financial support for population-based, organized screening programs for breast cancer. Health education and accessibility of screening services to women across the country should be strengthened, especially to women aged 50 years and above.

In 2020, breast cancer age-standardized incidence rates by world standard population ranged from $30 / 100,000$ in East Africa to above $95 / 100,000$ in Australia and New Zealand (4). Since the 1990s, breast cancer mortality notably declined in the developed world, which according to evidence of randomized controlled trials was largely due to breast cancer screening, though the actual amount of reduction attributable to screening was subject to controversy (5). By 2019, 121 countries have instituted a national screening program as a secondary prevention measure for breast cancer (6). To reduce the burden of breast cancer, China launched a national breast cancer screening program for rural female residents aged 35-59 years old in 2009 (extended to 35-64 years after 2012), as well as for urban female residents aged 40-69 years old in 2012 (later changed to 45-74 years after 2016) (7).

This study estimated the latest breast cancer screening rates in China based on nationally and provincially representative survey data. The survey was conducted in 2015 using a multistage, clusterrandomized sampling method. Female respondents aged 18 years and above were randomly selected from 298 districts/counties that were randomly selected from over 2,400 districts/counties across 31 provinciallevel administration divisions (PLADs). Respondents answered a set of questionnaires on chronic diseases and related behaviors, which were recorded by trained professionals from the local CDCs (8). With regard to breast cancer screening, all participants were asked whether they have ever had breast cancer screening, the time of the most recent screening, and the method of 
screening.

In 2015, of 88,250 households sampled, 100,543 female participants completed the survey from August to December, which yielded a $95.4 \%$ response rate. Several participants who did not meet criteria were excluded including 612 female participants who were less than 20 years, 7,902 participants who were unclear whether they have been screened, and 3,150 participants with missing data. The study was approved by the Ethical Committee of the National Center for Chronic and Non-Communicable Disease Control and Prevention, China CDC. All participants provided written informed consent.

Weighting was applied to all statistical analyses for both national and regional-specific estimates and the weighted proportion was reported. Chi-squared tests and logistic regression models were used to examine differences in unordered categorical variables and trends in ordered categorical variables, respectively. Taylor linearization methods with a finite population correction were used to estimate standard errors (SE). Statistical significance was determined as a two-sided $p<0.05$. All statistical analyses used SAS (version 9.4, SAS Institute Inc., Cary, USA).

The final sample of 88,879 female participants aged 20 years and above were 51.4 years of mean age, $59.0 \%$ from rural areas, and $41.3 \%$ with primary school or less education (Table 1).

The national breast cancer screening rate was $18.9 \%$ in women aged 20 years and above, and $25.7 \%$ in women aged 35-64 years. For women aged 20 years and above, the urban screening rate was significantly higher than the rural screening rate $(24.6 \%$ vs. $15.0 \%$, $p<0.0001)$. The eastern region showed a significantly higher screening rate than the central and western regions $(24.0 \%$ vs. $15.1 \%$ and $15.3 \%, p<0.0001)$. The 40-49 age group had the highest screening rate (29.2\%), whereas the 60-69 age group (12.3\%) and the 70 years and above age group (5.0\%) had much lower screening rates. Overall, $16.7 \%$ of women aged 20 years and above were screened within the past 2 years (Table 2). Moreover, $55.1 \%$ of women were screened by ultrasound, $14.5 \%$ by X-ray, $12.8 \%$ by only clinical examination, while $15.0 \%$ of women were unaware of their screening method.

Women with college or above education and with high school education (28.0\% and $24.7 \%$, respectively) had significantly higher screening rates than women with lower education levels $(p<0.0001)$. Women with higher income showed significantly higher screening
TABLE 1. Sociodemographic characteristics of female participants aged 20 years and above - China, 2015.

\begin{tabular}{|c|c|c|}
\hline Characteristics & $\begin{array}{c}\text { No. of } \\
\text { participants } \\
(\mathrm{N}=88,879)\end{array}$ & $\begin{array}{l}\text { Weighted } \\
\text { proportion } \\
(\%)(95 \% \mathrm{Cl})^{\star}\end{array}$ \\
\hline \multicolumn{3}{|l|}{ Age (years) } \\
\hline $20-29$ & 8,121 & $23.5(22.5-24.5)$ \\
\hline $30-39$ & 11,438 & $21.1(20.3-21.9)$ \\
\hline $40-49$ & 20,519 & $22.6(22.0-23.2)$ \\
\hline $50-59$ & 21,888 & $15.5(15.0-16.1)$ \\
\hline $60-69$ & 18,765 & $9.6(9.1-10.1)$ \\
\hline 70 and above & 8,148 & $7.7(6.9-8.5)$ \\
\hline \multicolumn{3}{|l|}{ Area type } \\
\hline Urban & 38,829 & $41.0(35.3-46.6)$ \\
\hline Rural & 50,050 & $59.0(53.4-64.7)$ \\
\hline \multicolumn{3}{|l|}{ Region } \\
\hline East & 33,603 & $42.5(38.6-46.5)$ \\
\hline Central & 25,669 & $32.2(28.5-35.9)$ \\
\hline West & 29,607 & $25.2(22.5-28.0)$ \\
\hline \multicolumn{3}{|l|}{ Education } \\
\hline Primary or less & 49,062 & $41.3(39.0-43.5)$ \\
\hline Junior high & 23,615 & $30.0(28.7-31.3)$ \\
\hline Senior high & 9,688 & $14.2(13.0-15.3)$ \\
\hline College or above & 6,514 & $14.5(12.7-16.3)$ \\
\hline
\end{tabular}

Household income per capita (CNY)

$\begin{array}{lcc}\text { Q1 }(<7,200) & 14,572 & 12.5(11.4-13.7) \\ \text { Q2 (7,200-14,999) } & 15,480 & 15.6(14.5-16.7) \\ \text { Q3 (15,000-24,999) } & 19,233 & 21.3(20.3-22.3) \\ \text { Q4 (25,000 and above) } & 24,168 & 32.5(30.2-34.9) \\ \text { Don't know/refused } & 15,426 & 18.0(16.5-19.6) \\ \text { Employment status } & & \\ \text { Employed } & 57,792 & 67.1(65.0-69.2) \\ \text { Housework } & 18,927 & 19.8(18.0-21.7) \\ \text { Retired } & 7,961 & 5.7(4.7-6.6) \\ \text { Unemployed } & 4,199 & 7.4(6.6-8.1) \\ \text { Health insurance coverage } & & \\ \text { No } & 2,476 & 4.1(3.7-4.6) \\ \text { Yes } & 86,403 & 95.9(95.4-96.4)\end{array}$

Health examination in the past 3 years

$\begin{array}{lll}\text { No } & 52,565 & 59.6(57.6-61.5) \\ \text { Yes } & 36,314 & 40.4(38.5-42.4) \\ \text { Self-assessed health status } & & \\ \text { Poor or fair } & 53,296 & 55.4(54.0-56.8) \\ \text { Good } & 35,583 & 44.6(43.2-46.0)\end{array}$

Proportions are weighted to represent the national total population with poststratification for age gender, and urban/rural residence. 


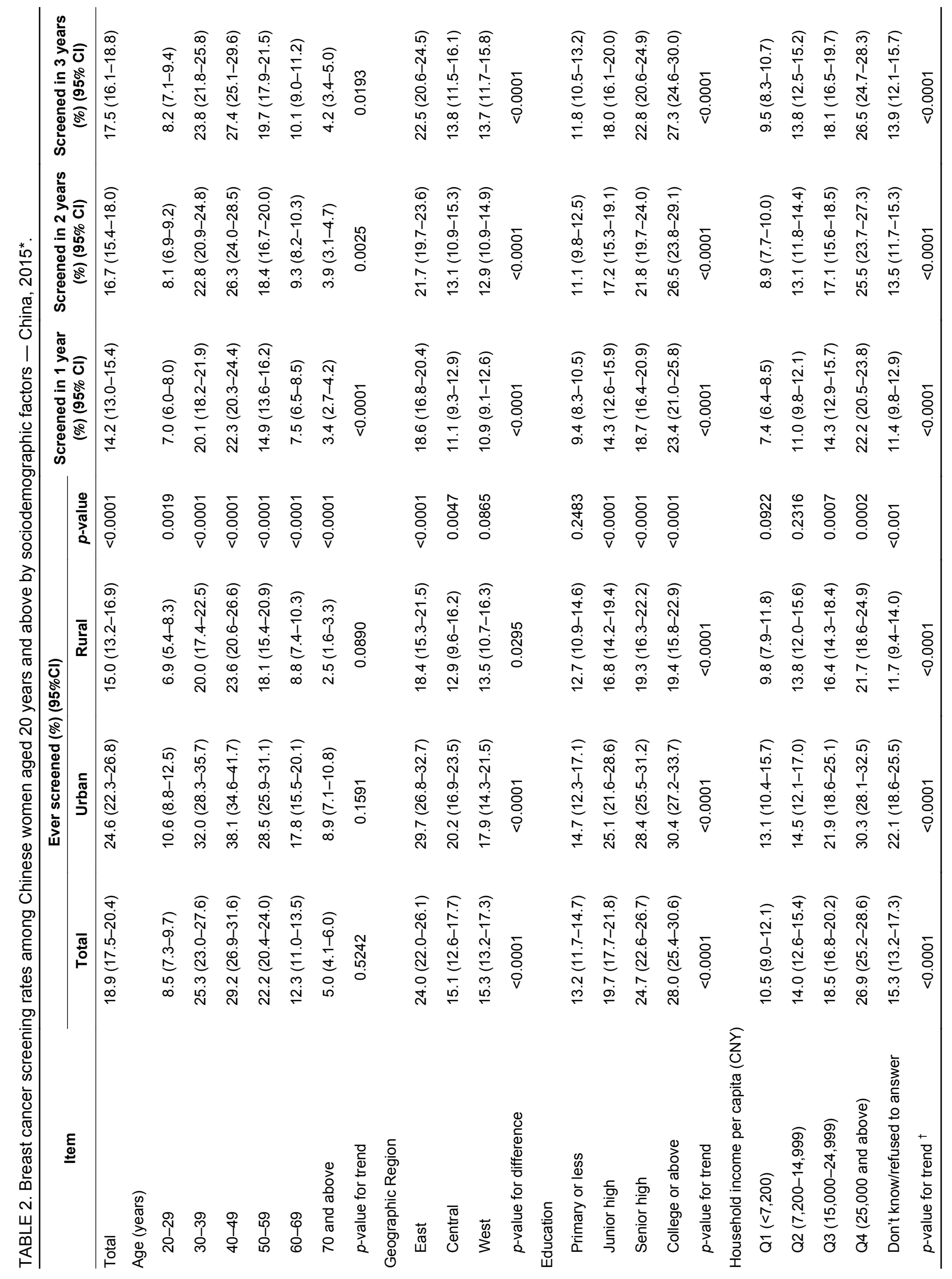




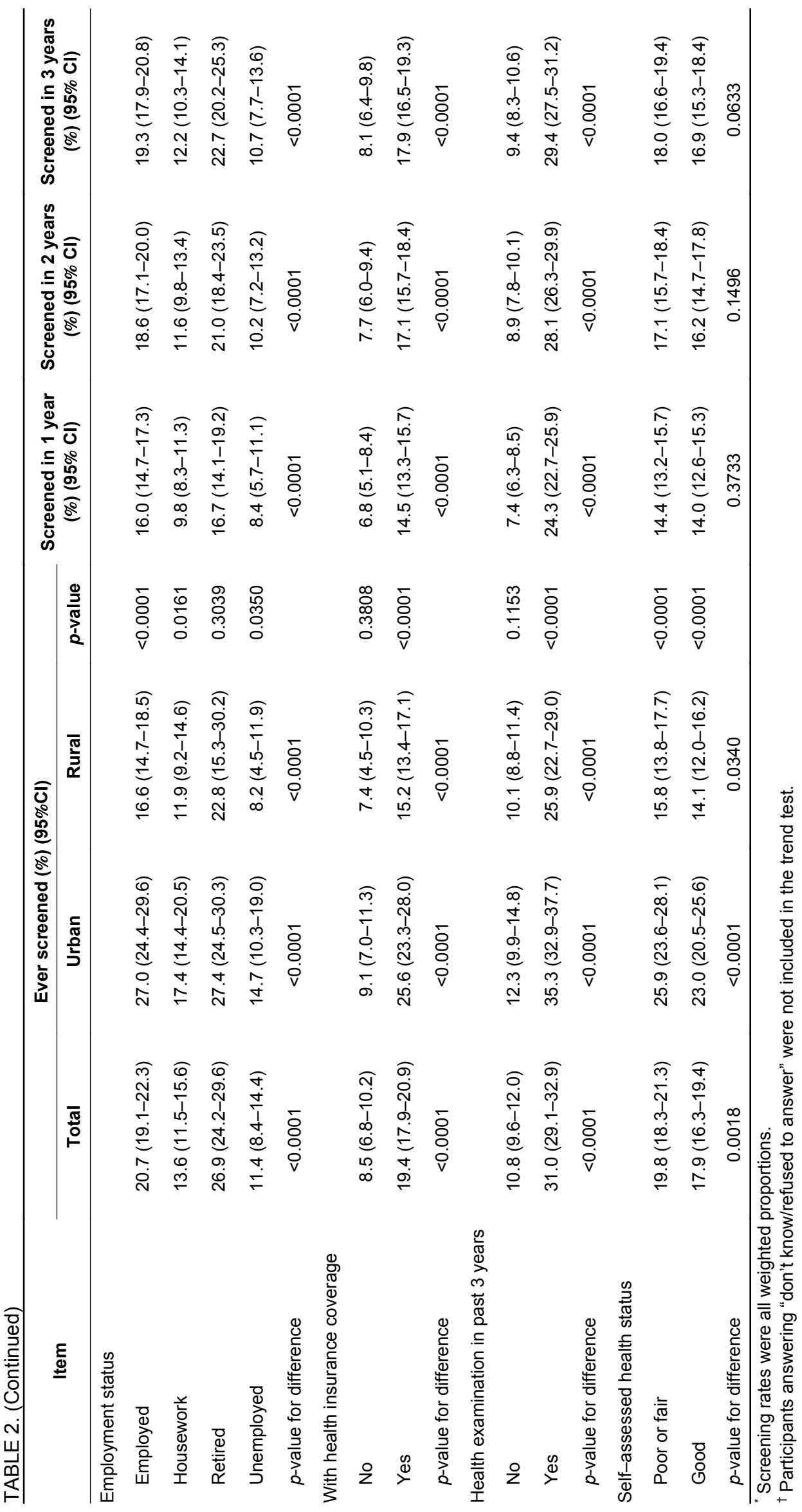


participation despite the fact that $15 \%$ of the participants did not reveal their income level. Retired women (26.9\%) had a significantly higher screening rate than employed women, women doing housework, and unemployed women $(p<0.0001)$.

Women with health insurance had significantly higher screening participation than women without health insurance $(19.4 \%$ vs. $8.5 \%, p<0.0001)$. Women with health check-ups in the past 3 years had significantly higher screening rates than women without health check-ups $(31.0 \%$ vs. $10.8 \%$, $p<0.0001)$. Women with self-assessed poor or fair health status had statistically significantly higher screening rates than women with self-assessed good health status $(19.8 \%$ vs. $17.9 \%, p=0.0018)$.

Provincial data revealed disparities in screening rates across the 31 PLADs. The screening rates in Beijing and Shanghai exceeded $40 \%$, whereas Xizang (Tibet), Anhui, and Hebei had the lowest screening rates of less than $10 \%$ (Figure 1).

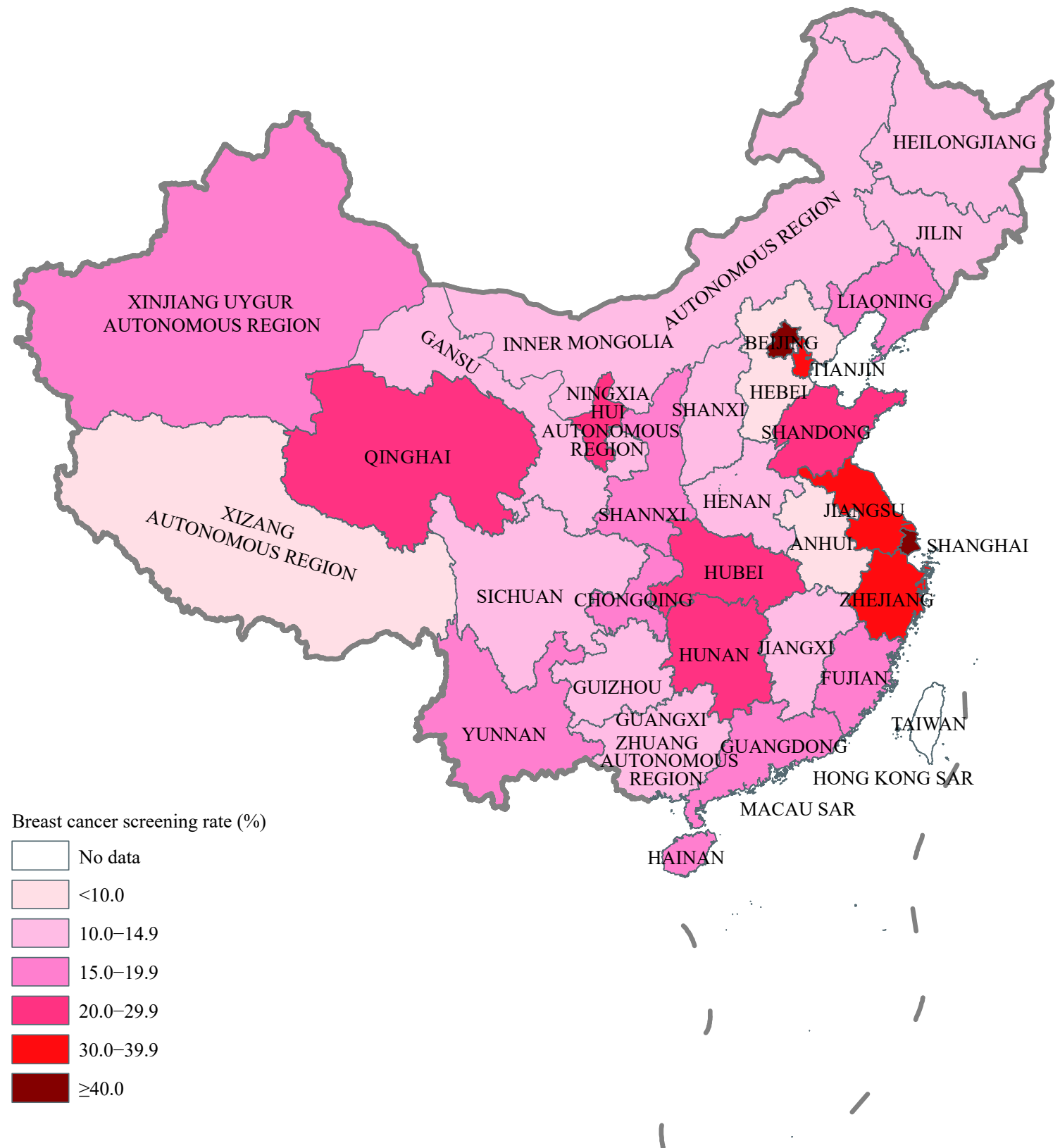

FIGURE 1. Breast cancer screening rates among Chinese women aged 20 years and above at the provincial level in China, 2015. 


\section{DISCUSSION}

This study showed that breast cancer screening rates in China in 2015 reached $18.9 \%$ and $25.7 \%$ for women aged 20 years and above and women aged 35-64 years, respectively. While the screening uptake for all adult women declined about 3\% from 2010 to 2015 (2), the screening rate for women aged 35-64 years increased about 3\% from 2013 to 2015 (3), which indicated positive influence of the national breast cancer screening program. Nevertheless, screening uptake remained low compared to screening rates of above $50 \%$ in developed countries ( $\sigma$ ). Continued efforts should be made to strengthen breast cancer screening uptake in China.

This study found that the group aged 30-49 years had the highest screening rates, whereas the 60 years and above age groups had ever screening rates of lower than $15 \%$ and screening in the past two years lower than $10 \%$. Such age distribution of screening peaked earlier than recommended. While most international breast cancer screening guidelines recommended biennial screening for the 50-74 years age group, the latest guidelines released in 2019 in China based on Chinese women's risk profile recommended biennial screening for the 45-69 years age group of common risk, as well as for women aged 40-44 years and women aged 69 years and above with more than 10 years of life expectancy of common risk upon individual will, whereas women under 40 years old were not recommended for regular screening (9).

This study also found that the rural areas and the central and western regions still lagged far behind the urban areas and the eastern region in breast cancer screening uptake, despite national free provision of screening services to women in rural areas. In addition, women with lower education and lower income levels had significantly lower screening participation. These findings were consistent with existing literature that individual and area socioeconomic status (SES) were positively associated with cancer screening participation (10). Moreover, the results revealed that participation in breast cancer screening was significantly lower than cervical cancer screening (8) upon free screening services. The results highlighted the importance to identify barriers to breast cancer screening, such as embarrassment, as well as the importance of strengthening health education for low SES groups to improve breast cancer screening participation.

This study further found that health insurance was positively associated with screening uptake, which echoes existing literature that health insurance coverage improves access to cancer screening.

This study was subject to some limitations. The absence of some data due to lack of subpar attendance limited the conclusions of the analysis. Moreover, this study was limited by response bias as $7.8 \%$ of the initial respondents were excluded from the final analysis, who were unclear of their screening history and were slightly older, more rural, and less educated than the final sample. Assuming that these respondents were all unscreened, it would lower final screening rates by about $1 \%-2 \%$, which would not change the conclusion. Recall bias may also occur as the respondents might incorrectly recall their screening history.

In conclusion, this study provides the largest nationwide and population-based self-reported history of breast cancer screening in China in 2015. Nearly one-fifth of Chinese women ever had breast cancer screening. Continued efforts should be made to strengthen national and local policy initiatives and financial support for population-based, organized screening programs for breast cancer, and strengthen health education and accessibility of screening services to women across the country, especially women aged 50 years and above.

Funding: National Key Research and Development Program of China (2016YFC0901300, 2016YFC 0901301).

doi: $10.46234 /$ ccdcw2021.078

\# Corresponding authors: Linhong Wang, wanglinhong@ncncd. chinacdc.cn; Xiaoying Zheng, xzheng@pku.edu.cn.

\footnotetext{
1 National Center for Chronic and Non-Communicable Disease Control and Prevention, Chinese Center for Disease Control and Prevention, Beijing, China; ${ }^{2}$ Institute of Population Research, Peking University, Beijing, China; ${ }^{3}$ Department of Maternal and Child Health, School of Public Health, Peking University, Beijing, China.

\& Joint first authors.
}

Submitted: March 03, 2021; Accepted: March 24, 2021

\section{REFERENCES}

1. Zheng RS, Sun KX, Zhang SW, Zeng HM, Zou XN, Chen R, et al. Report of cancer epidemiology in China, 2015. Chin J Oncol 2019;41(1):19 - 28. http://dx.doi.org/10.3760/cma.j.issn.0253-3766. 2019.01.008. (In Chinese).

2. Wang BH, He MF, Wang LM, Engelgau MM, Zhao WH, Wang LH. Breast cancer screening among adult women in China, 2010. Prev Chronic Dis 2013;10:E183. http://dx.doi.org/10.5888/pcd10.130136.

3. Bao HL, Wang LH, Wang LM, Fang LW, Zhang M, Zhao ZP, et al. Study on the coverage of cervical and breast cancer screening among women aged 35-69 years and related impact of socioeconomic factors in China, 2013. Chin J Epidemiol 2018;39(2):208-12. http://dx. 
doi.org/10.3760/cma.j.issn.0254-6450.2018.02.014. (In Chinese).

4. Globocan 2020. Breast cancer fact sheet. https:/gco.iarc.fr/today/data/ factsheets/cancers/20-Breast-fact-sheet.pdf. [2021-02-26].

5. Nelson HD, Humphrey LL, Fu RW. Estimates of screening benefit: the randomized trials of breast cancer screening. In: Houssami N, Miglioretti D, editors. Breast cancer screening: an examination of scientific evidence. Amsterdam: Elsevier. 2016; p. 29-49. http://dx. doi.org/10.1016/B978-0-12-802209-2.00002-4.

6. Globocan 2020. Primary and secondary prevention of cancer: response by country. https://apps.who.int/gho/data/view.main.24766. [2021-02-26].

7. Chen WQ, Li N, Shi JF, Ren JS, Chen HD, Li J, et al. Progress of cancer screening program in urban China. Chin Cancer 2019;28(1): 23 - 5. http://dx.doi.org/10.11735/j.issn.1004-0242.2019.01.A003.
(In Chinese).

8. Zhang M, Zhong YJ, Zhao ZP, Huang ZJ, Zhang X, Li C, et al. Preplanned studies: cervical cancer screening rates among Chinese women - China, 2015. China CDC Wkly 2020;2(26):481 - 6. http://dx.doi.org/10.46234/ccdcw2020.128.

9. Huang YB. China breast cancer screening guidelines for women. Chin J Clin Oncol 2019;46(9):429 - 31. http://dx.doi.org/10.3969/j.issn. 1000-8179.2019.09572. (In Chinese).

10. Pruitt SL, Shim MJ, Mullen PD, Vernon SW, Amick III BC. Association of area socioeconomic status and breast, cervical, and colorectal cancer screening: a systematic review. Cancer Epidemiol Biomarkers Prev 2009;18(10):2579 - 99. http://dx.doi.org/10.1158/ 1055-9965.EPI-09-0135. 